Revista Investigaciones y Aplicaciones

Nucleares, 5, 5-13, 2021

Recibido: 7 de mayo de 2021

Aceptado: 5 de septiembre de 2021

Publicado en línea: 28 de octubre de 2021

Doi: https://doi.org/10.32685/2590-7468/

invapnuclear.5.2021.579

\section{(c) (i)}

Esta obra está bajo licencia internacional Creative Commons Reconocimiento 4.0.

\title{
Ajustes metodológicos desarrollados durante los ejercicios de intercomparación para la determinación de emisores gamma en muestras ambientales
}

\section{Development of methodological modifications during intercomparison exercises to detect gamma emitters in environmental samples}

Giovanni Vela ${ }^{1}$, Fernando Mosos $^{1}$, Leidy Tatiana Ávila ${ }^{1}$

Citación: G. Vela Guzmán, F. Mosos y L. T. Ávila, “Ajustes metodológicos desarrollados durante los ejercicios de intercomparación para la determinación de emisores gamma en muestras ambientales", Revista Investigaciones y Aplicaciones Nucleares, n. ${ }^{\circ}$ 5, pp. 5-13, 2021. https://doi.org/10.32685/2590-

7468/invapnuclear.5.2021.579

\section{Resumen}

El Laboratorio de Radiometría Ambiental (LRA) ha participado en los ensayos de aptitud realizados por el Organismo Internacional de Energía Atómica (OIEA) desde el año 2015, como parte de su proceso de aseguramiento de la calidad analítica para la técnica de espectrometría de rayos gamma. El presente trabajo da a conocer los resultados obtenidos por el LRA mediante la técnica de espectrometría de rayos gamma, durante sus seis años de participación, diferenciados según tipo de muestra y radionucleidos objeto de análisis. Se presenta la descripción de los criterios de calificación de los ensayos de aptitud entre laboratorios y su relación con la validación del método, así como las mejoras implementadas para garantizar la validez de los resultados.

Palabras clave: espectrometría de rayos gamma, ensayo de aptitud, validación del método, criterios de calificación, aseguramiento de la calidad analítica.

\section{Abstract}

The Environmental Radiometry Laboratory (LRA in Spanish) has participated in proficiency tests carried out by the International Atomic Energy Agency (IAEA) since 2015 as part of an analytical quality assurance process. The results obtained by the LRA using the gamma-ray spectrometry technique over six years of participation are presented and classified

${ }^{1}$ Dirección de Asuntos Nucleares, Servicio Geológico Colombiano, Bogotá, Colombia. Autor de correspondencia: Giovanni Vela, gvela@sgc.gov.co 
according to the type of sample and analyzed radionuclides. The qualification criteria used for the proficiency tests for the laboratories and how these criteria affected the validation of the method being tested are presented, along with a description of improvements that were implemented to guarantee the validity of the results.

Keywords: gamma-ray spectrometry, proficiency test, method validation, qualification criteria, analytical quality assurance.

\section{Introducción}

$\mathbf{E}$ 1 objetivo de este trabajo es ampliar metodológicamente los criterios de control interno y ajustar los parámetros operacionales que permitan tanto la determinación de bajas concentraciones de emisores gamma en matrices ambientales, como la identificación y cuantificación de radionucleidos por medio de una respuesta rápida. Para esto, se optimizó el tiempo de análisis, se mejoró el límite inferior de detección (LID) para los diferentes detectores del laboratorio, que se calificaron por medio de las herramientas estadísticas de evaluación de desempeño de los ejercicios de intercomparación en términos de reproducibilidad, el instrumento de cálculo MATLAB para la determinación matemática de las correcciones complejas del decaimiento radiactivo, y las correcciones a la eficiencia conforme la herramienta de simulación de Canberra Industries Laboratory Sourceless Calibration Software (LABSOCs).

La reproducibilidad y calidad de los resultados obtenidos en un laboratorio de ensayo se establecen conforme a aquellos derivados de los criterios de aceptación que califican la exactitud y precisión de la medición. La competencia del laboratorio se determina mediante la validación del método de ensayo, la participación en programas de ensayos de aptitud [1], el ajuste de los controles de calidad internos establecidos y la calificación de su sistema de calidad, basado en la norma internacional ISO-IEC 17025 [2]. Los resultados de desempeño en los ensayos de aptitud son considerados la actividad más concluyente para calificar la calidad de todos los procesos asociados al desarrollo del método de medición [3], [4].

El Laboratorio de Radiometría Ambiental (LRA) del Servicio Geológico Colombiano ha participado, desde el año 2015, en el programa de intercomparación de la red de laboratorios de ensayo para la medición de la radiactividad ambiental (ALMERA), orientado a la medición de muestras con un bajo contenido de radiactividad. Esta actividad ha permitido al LRA alcanzar la calidad requerida y las mejo- ras significativas que demuestran la evolución asociada a su madurez metodológica a través de la evaluación de los datos analíticos.

\section{Materiales y métodos}

\subsection{Detectores}

Los sistemas de espectrometría empleados para el análisis de las muestras objetivo durante los diferentes ejercicios de intercomparación en los que ha participado el LRA se describen en la tabla 1.

\subsection{Materiales de referencia certificados}

Los materiales de referencia empleados para las calibraciones de energía en función del canal y de la eficiencia para cada uno de los detectores se presentan en la tabla 2.

\subsection{Calibración de la eficiencia en función de la energía}

Los criterios seleccionados para identificar la(s) causa(s) raíz de un resultado como no satisfactorio fueron:

» Determinación de factores de corrección asociados a la eficiencia de cada detector.

\Estimación y calificación de la incertidumbre obtenida por herramientas computacionales (Genie2000 v3.4).

» Características del detector y desempeño operacional.

»Determinación de la relación eficiencia vs. energía por herramienta de simulación y modelo matemático manual.

i. Herramienta de simulación. El programa de Canberra Industries Laboratory Sourceless Object Calibration - Software (LabSOCS) es una herramienta computacional que realiza calibraciones matemáticas de eficiencia para detectores de Ge, sin el uso de fuentes radiactivas [1]. Esto permite realizar calibraciones rápidas y precisas de muchas matrices considerando la complejidad de su composición, en diferentes geometrías de análisis. Mediante este pro- 
Tabla 1. Características de los sistemas espectrométricos empleados en los ensayos de aptitud entre laboratorios de radiactividad ambiental

\begin{tabular}{|c|c|c|}
\hline Código interno del detector & Características & Uso en ejercicio de intercomparación \\
\hline DET01 & $\begin{array}{l}\text { HPGe modelo CANBERRA GC7020. Detector tipo p. Eficiencia relativa } \geq 70 \% \text {. Resolución (FWHM), } \\
\text { energía de } 1332,6 \mathrm{keV} \mathrm{del}{ }^{60} \mathrm{Co} \leq 2,0 \mathrm{keV} \text {. Rango energético de trabajo } 40-2000 \mathrm{keV} \text {. }\end{array}$ & $2016,2017,2018$ \\
\hline DET02 & $\begin{array}{l}\text { HPGe modelo CANBERRA GX5019. Detector tipo n. Eficiencia relativa } \geq 50 \% \text {. Resolución (FWHM), } \\
\text { energía de } 1332,6 \mathrm{keV} \mathrm{del}{ }^{60} \mathrm{Co} \leq 1,9 \mathrm{keV} \text {. Rango energético de trabajo } 10-2000 \mathrm{keV} \text {. }\end{array}$ & 2019,2020 \\
\hline DET03 & $\begin{array}{l}\text { HPGe modelo ORTEC GEM 10195. Detector tipo p. Eficiencia relativa } \geq 30 \% \text {. Resolución (FWHM), } \\
\text { energía de } 1332,6 \mathrm{keV} \text { del }{ }^{60} \mathrm{Co} \leq 2,0 \mathrm{keV} \text {. Rango energético de trabajo } 40-2000 \mathrm{keV} \text {. }\end{array}$ & 2015,2016 \\
\hline
\end{tabular}

Tabla 2. Materiales de referencia certificados empleados en la calibración de energía y eficiencia

\begin{tabular}{|c|c|c|c|c|c|c|c|}
\hline \multirow[b]{2}{*}{$\begin{array}{l}\text { Material de } \\
\text { referencia }\end{array}$} & \multirow[b]{2}{*}{ Tipo } & \multirow[b]{2}{*}{ Radionucleido } & \multirow[b]{2}{*}{$\begin{array}{c}\text { Energía } \\
\text { keV }\end{array}$} & \multirow[b]{2}{*}{ Intensidad (\%) } & \multirow[b]{2}{*}{$\begin{array}{c}\text { Densidad } \\
\left(\mathrm{g} \mathrm{cm}^{-3}\right)\end{array}$} & Actividad & \multirow[b]{2}{*}{ Fecha de ref. (1) } \\
\hline & & & & & & $\begin{array}{c}\mathbf{A} \\
\mathbf{B q} \mathbf{~ k g}^{-1}(1)\end{array}$ & \\
\hline \multirow{2}{*}{ EU155/NA22 } & \multirow{2}{*}{ Puntual } & ${ }^{155} \mathrm{Eu}$ & $86,5479(10)$ & $30,7(3)$ & \multirow[b]{2}{*}{$=$} & $37000 \pm 740$ & \multirow{2}{*}{$30 / 10 / 2017$} \\
\hline & & ${ }^{22} \mathrm{Na}$ & $1274,537(7)$ & $99,94(13)$ & & $37000 \pm 740$ & \\
\hline \multirow{4}{*}{ MGS-2 } & \multirow{4}{*}{ Puntual } & ${ }^{125} \mathrm{Sb}$ & $427,874(4)$ & $29,55(24)$ & \multirow{4}{*}{$=$} & $12158 \pm 608$ & \multirow{4}{*}{$19 / 01 / 2008$} \\
\hline & & ${ }^{155} \mathrm{Eu}$ & $86,5479(10)$ & $30,7(3)$ & & $5956 \pm 298$ & \\
\hline & & ${ }^{54} \mathrm{Mn}$ & $834,848(3)$ & $99,9752(5)$ & & $23147 \pm 1157$ & \\
\hline & & ${ }^{60} \mathrm{Co}$ & $1332,492(4)$ & $99,9826(6)$ & & $5553 \pm 278$ & \\
\hline \multirow{5}{*}{ IAEA - RGTh - 1} & \multirow{5}{*}{ Matriz sólida - Thorium Ore } & ${ }^{232} \mathrm{Th}$ & $238,632(2)$ & $43,6(5)$ & \multirow{5}{*}{$\underline{1,54}$} & $3250 \pm 90$ & \multirow{5}{*}{$1 / 01 / 1987$} \\
\hline & & \multirow{2}{*}{${ }^{235} \mathrm{U}$} & $185,720(4)$ & $57,0(3)$ & & $3,6 \pm 0,3$ & \\
\hline & & & $143,767(3)$ & $10,94(6)$ & & $3,6 \pm 0,3$ & \\
\hline & & ${ }^{238} \mathrm{U}$ & $351,932(2)$ & $35,60(7)$ & & $78 \pm 6$ & \\
\hline & & ${ }^{40} \mathrm{~K}$ & $1460,822(6)$ & $10,55(11)$ & & $6,3 \pm 3,2$ & \\
\hline \multirow{3}{*}{ IAEA - RGU - 1} & \multirow{3}{*}{ Matríz sólida - Uranium Ore } & ${ }^{235} \mathrm{U}$ & $185,720(4)$ & $57,0(3)$ & \multirow{3}{*}{$\underline{1,59}$} & $228 \pm 4$ & \multirow{3}{*}{$1 / 01 / 1987$} \\
\hline & & & $143,767(3)$ & $10,94(6)$ & & $228 \pm 4$ & \\
\hline & & ${ }^{238} \mathrm{U}$ & $351,932(2)$ & $35,60(7)$ & & $4940 \pm 60$ & \\
\hline
\end{tabular}

(1) Información suministrada en el certificado de análisis del material de referencia.

cedimiento se calcula la curva de eficiencia asociada a partir de las características propias y específicas del detector, la geometría de análisis, la composición química de la muestra y su densidad aparente. El ajuste de eficiencia de In-Situ Object Calibration Software (ISOCS) emplea el código Montecarlo N-Particle (MCNP) que simula el transporte de partículas a través de la materia [2], basado en eficiencias de referencia obtenidas experimentalmente con fuentes puntuales trazables al National Institute of Standards and Technology (NIST). El programa genera una cuadrícula que permite simular la eficiencia con su respectiva incertidumbre en cualquier energía entre 45 y $7000 \mathrm{keV}$, empleando interpolación parabólica entre las eficiencias de referencia [6], [7]. Los efectos de sumas en cascada son corregidos por el software Genie ${ }^{\text {m }}$ 2000, y sus algoritmos están patentados por Canberra (patente de EE. UU. 6225634).

ii. Modelo manual. Para los detectores DET01 y DET03 (tabla 1), el LRA desarrolló dos hojas de cálculo ma- nual mediante las que se determinó la eficiencia en las energías características de cada radionucleido presente en los MRC. Los factores de corrección por picos suma son determinados experimentalmente empleando fuentes puntuales certificadas en diferentes posiciones de distancia fuente-detector [8]. El efecto causado por la densidad de la muestra se resolvió por la construcción de una curva de eficiencia con MRC de densidad cercana o similar a la de la muestra objetivo. Para las matrices líquidas, este procedimiento fue sencillo (misma densidad); en las matrices sólidas, generó fuertes desviaciones en los resultados finales debido a la diferencia entre las densidades del MRC y las muestras. Este modelo no es exacto y es bastante costoso en tiempo y materiales.

\subsection{Preparación de muestras}

Las muestras objetivo fueron gestionadas conforme al proceso de prestación del servicio establecido. El acondicionamiento de estas consistió en el transvase a la geometría in- 
terna de análisis y el registro de peso neto hasta el volumen de $100 \mathrm{ml}$. La geometría empleada en el laboratorio es un envase cilíndrico de polietileno de alta densidad (PE-HD), presentada en la figura 1. Las muestras se ubicaron en contacto con el detector.

Se garantizó la hermeticidad de las muestras, por la misma configuración de selle de la geometría y por el uso adicional de una bolsa de selle hermético, en donde se introdujo la geometría de análisis. Para estimar la humedad en las matrices sólidas, se evaluó la pérdida de peso a una porción de muestra de $5 \mathrm{~g}$ aproximadamente. Esta se mantuvo a $105^{\circ} \mathrm{C}$ durante 24 horas, sin circulación de aire.

La preparación de la geometría de filtros consistió en recortar el pliego de papel en los extremos indicados por el protocolo y almacenarla en bolsas de selle hermético apropiadas para el tamaño de la muestra.

El tiempo de análisis de las muestras se determinó conforme las indicaciones suministradas por el OIEA, en cada uno de los ensayos de aptitud, asegurando una estadística para un nivel de confianza superior a $90 \%$.
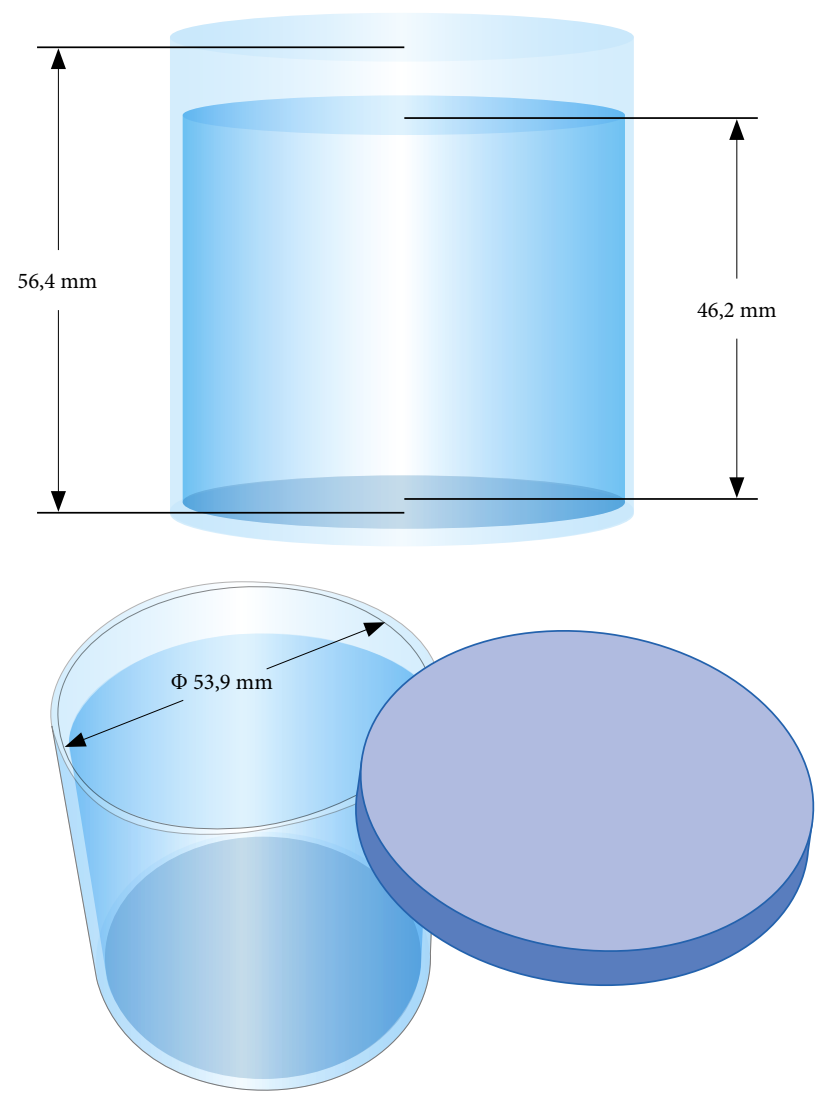

Figura 1. Geometría interna de análisis LRA

\subsection{Adquisición de blancos}

Se analizaron blancos por un tiempo de análisis igual al establecido para las muestras objetivo. Para las muestras líquidas, se utilizó agua destilada acidulada con ácido clorhídrico $(\mathrm{HCl})$ hasta $\mathrm{pH}=2$. Para las muestras sólidas de densidad mayor a $1,0 \mathrm{~g} \mathrm{~cm}^{-3}$, se trabajó solución acuosa de heteropulitungstato de litio con densidad de $1,45 \mathrm{~g} \mathrm{~cm}^{-3}$. Para las muestras en geometría de filtros, se empleó un blanco de papel bond con densidad $0,75 \mathrm{~g} \mathrm{~cm}^{-3}$.

\subsection{Descripción de la calificación ensayos de aptitud entre laboratorios}

Los ensayos de aptitud llevados a cabo por el programa de evaluación de desempeño ALMERA (Analytical Laboratories for the Measurement of Environmental Radioactivity) del Organismo Internacional de Energía Atómica (OIEA), y en el cual participa activamente el LRA, califican los siguientes criterios:

\ El sesgo relativo entre los valores reportados y objetivo (la mejor estimación del valor real): el sesgo relativo es comparado con el máximo sesgo relativo aceptado (MARB, por su sigla en inglés) el cual es determinado para cada mensurando a partir de los antecedentes físicos de los métodos radioanalíticos, incluido el nivel de radiactividad y la complejidad de la tarea (figura 2). Si el sesgo relativo Bias relative $\leq \mathrm{MARB}$ el resultado es aceptado para exactitud.

\) Basado en el principio de adecuación al propósito, las buenas prácticas de laboratorio y la incertidumbre relativa combinada expandida, se determina el estadístico $\mathrm{P}$. Si se cumple que $\mathrm{P} \leq \mathrm{MARB}$ y Bias $_{\text {relative }} \leq \mathrm{k} \star \mathrm{P}$, el resultado es aceptado para precisión. Si uno de ellos no se cumple, el resultado será de no aceptado para precisión (figura 2). $k$ es el factor de cobertura para un nivel de confianza del $95 \%$ y su valor es de 2,65. Si el resultado reportado está dentro del valor +/- MARB, pero no se superpone con el valor objetivo dentro de sus incertidumbres, esta ecuación permite decidir si es o no significantemente diferente.

El estadístico $z$ incluye la desviación estándar robusta sin afectaciones. Esta se deriva de los resultados de los reportes y, en consecuencia, es influenciada por el desempeño de los participantes (figura 2). 
La calificación final dada por el programa ALMERA indica:

$\gg$

Aceptado, cuando exactitud y precisión logran el estatus de aceptado.

$\gg$

》)

Alerta, cuando la exactitud es aceptada, pero la precisión es no aceptada.

\subsection{Descripción del criterio de madurez}

El LRA estableció el criterio de madurez con base en el consolidado de calificaciones obtenido, durante su participación en los ensayos de aptitud de ALMERA. La calificación consiste en la correlación entre el número de veces que cada radionucleido objetivo ha sido evaluado en todas las matrices, frente al número de veces que ha sido calificado como aceptado para el LRA.

Se fijó la calificación madurez metodológica para aquellos radionucleidos en los que el laboratorio obtuvo aceptado en el $50 \%$ o más de sus participaciones en los ensayos de aptitud; en proceso y con avances, cuando se alcanzó entre el $20 \%$ y $50 \%$, y “por desarrollar", si en los resultados obtenidos la calificación de aceptable ha sido inferior al $20 \%$.

\section{Resultados y discusión}

En esta sección se presenta el resumen de los resultados obtenidos en las diferentes participaciones en los ensayos de aptitud, por parte del LRA, desde el año 2015.

La determinación de actividades y concentraciones de actividad de los radionucleidos se puede realizar con varias de sus energías características. Para la generación del reporte se seleccionó la energía que presentaba mayor probabilidad de emisión o rendimiento gamma; también, se consideró que la energía seleccionada no se encuentre afectada por la emisión de fotones de otro radionucleido presente en la muestra. En el caso de las cadenas radiactivas, se tomó el fotopico de la hija que presentó la mejor estadística en área de interés (cuentas), una vez logrado el equilibrio secular.

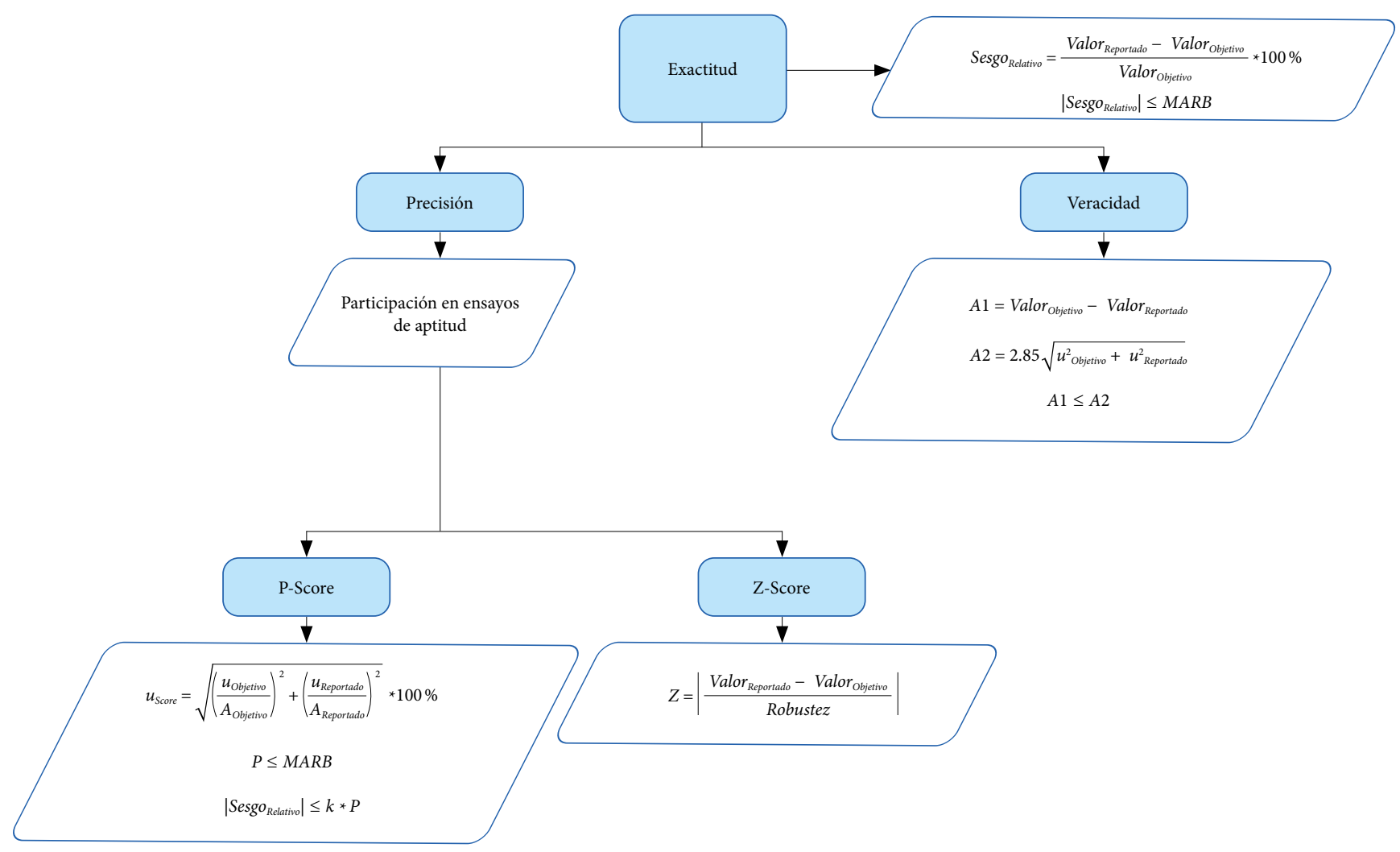

Figura 2. Diagrama de flujo de los criterios de calificación empleados en los ensayos de aptitud desarrollados por el Organismo Internacional de Energía Atómica 
La tabla 3 presenta el resumen de los resultados obtenidos por el LRA durante su participación en los ensayos de aptitud IAEA-TEL-ALMERA, entre los años 2015 a 2020, indicando la cantidad de radionucleidos en los que el laboratorio obtuvo resultados de aceptado, no aceptado, alerta y no reportados.

La tabla 4 presenta el resumen de los resultados obtenidos por el LRA, en sus participaciones en las pruebas de desempeño entre laboratorios, considerando los radionucleidos evaluados, el tipo de matriz y la madurez metodológica del laboratorio.

Los valores reportados de algunos de los radionucleidos objetivo por diferentes laboratorios participantes corresponden al uso de diferentes técnicas radiactivas, como espectrometría alfa, espectrometría gamma, centelleo líquido, entre otros. El LRA reporta todas las actividades de los radionucleidos emisores gamma, empleando exclusivamente la espectrometría de rayos gamma con detectores de germanio ultrapuro (GeHp).
La evaluación de las causas raíz en los casos de radionucleidos con calificaciones de no reportado, no aceptado $\mathrm{y}$ en alerta, permitió adelantar mejoras en diferentes procedimientos, como el selle hermético de la geometría de análisis, la definición de ventanas de tiempo analítico para las series naturales y la corrección de actividad mediante la solución de las ecuaciones de Bateman. Dentro de las acciones se encuentra la definición de ventanas de tiempo analítico presentadas en la tabla 4. Debido a que, en las series naturales del $\mathrm{U} y \mathrm{Th}$, los emisores de vida larga como ${ }^{238} \mathrm{U},{ }^{234} \mathrm{U},{ }^{230} \mathrm{Th}$, ${ }^{226} \mathrm{Ra}$, no se pueden determinar directamente, se seleccionan las hijas de vida corta que tienen emisiones gamma con una intensidad de emisión gamma suficiente.

La tabla 5 presenta el consolidado de los resultados obtenidos por el laboratorio, en su participación en los ensayos de aptitud ALMERA, así como el criterio de madurez.

Tabla 3. Resumen participaciones LRA en IAEA-TEL-ALMERA entre los años 2015 a 2020

\begin{tabular}{|c|c|c|c|c|c|c|}
\hline Radionucleidos & PT-2015 & PT-2016 & PT-2017 & PT-2018 & PT-2019 & PT-2020 \\
\hline No reportados & $60 \%$ & $29 \%$ & $40 \%$ & $50 \%$ & $41 \%$ & 0 \\
\hline Aceptados & $19 \%$ & $14 \%$ & $54 \%$ & $26 \%$ & $41 \%$ & $100 \%$ \\
\hline Alerta & 0 & 0 & 0 & $4 \%$ & $9 \%$ & 0 \\
\hline No aceptados & $21 \%$ & $57 \%$ & $6 \%$ & $20 \%$ & $9 \%$ & 0 \\
\hline Total & 27 & 7 & 15 & 46 & 32 & 30 \\
\hline
\end{tabular}

Tabla 4. Ruta crítica temporal para análisis de las series naturales de ${ }^{232} \mathrm{Th}$ y ${ }^{238} \mathrm{U}$

\begin{tabular}{|c|c|c|c|}
\hline Radionucleido objetivo & Radionucleido análisis & Ventana de tiempo & Comentarios \\
\hline- & - & Día 0 & $\begin{array}{l}\text { Preparación y acondicionamiento de la muestra objetivo en geometría de } \\
\text { análisis. }\end{array}$ \\
\hline${ }^{228} \mathrm{Ra}$ & ${ }^{228} \mathrm{Ac}$ & A partir del día 2 & \multirow{3}{*}{$\begin{array}{l}\text { Serie natural del }{ }^{232} \mathrm{Th} \text {. Siempre asegurar hermeticidad en la geometría de } \\
\text { análisis. }\end{array}$} \\
\hline${ }^{220} \mathrm{Rn}-{ }^{224} \mathrm{Ra}-{ }^{208} \mathrm{Tl}$ & ${ }^{212} \mathrm{~Pb}-{ }^{212} \mathrm{Bi}$ & Entre los días 2 al 5 & \\
\hline${ }^{228} \mathrm{Th}$ & ${ }^{212} \mathrm{~Pb}-{ }^{212} \mathrm{Bi}$ & A partir del día 25 & \\
\hline${ }^{226} \mathrm{Ra}$ & ${ }^{214} \mathrm{~Pb}-{ }^{214} \mathrm{Bi}$ & A partir del día 25 & \multirow{2}{*}{$\begin{array}{c}\text { Serie natural del }{ }^{238} \mathrm{U} \text {. Siempre asegurar hermeticidad en la geometría de } \\
\text { análisis. }\end{array}$} \\
\hline${ }^{222} \mathrm{Rn}$ & ${ }^{214} \mathrm{~Pb}-{ }^{214} \mathrm{Bi}$ & Entre $4-48$ horas & \\
\hline
\end{tabular}


Tabla 5. Resumen participaciones LRA en IAEA-TEL-ALMERA entre los años 2015 a 2020

\begin{tabular}{|c|c|c|c|c|c|c|c|c|c|c|c|c|c|c|c|c|c|c|}
\hline \multirow{3}{*}{\multicolumn{2}{|c|}{$\begin{array}{l}\text { Radionucleidos evaluados } \\
\text { Proficiency Test }\end{array}$}} & \multirow{4}{*}{$\begin{array}{c}\begin{array}{c}\text { Criterio madurez } \\
\text { LRA }\end{array} \\
\text { M. M. } \\
\end{array}$} & \multicolumn{16}{|c|}{ Tipo de matriz } \\
\hline & & & \multicolumn{7}{|c|}{ Líquida } & \multicolumn{6}{|c|}{ Sólida } & \multicolumn{3}{|c|}{ Filtros } \\
\hline & & & \multirow{2}{*}{2015} & \multirow{2}{*}{2016} & \multirow[t]{2}{*}{2017} & & 201 & & 2020 & 2015 & 2016 & 2017 & 2018 & 2019 & 2020 & 2019 & & 2020 \\
\hline${ }^{100 \mathrm{~m}} \mathrm{Ag}$ & $\gamma$ & & & & & $\mathrm{X}$ & & & & & & & & & & & & A $\mathbf{A}$ \\
\hline${ }^{241} \mathrm{Am}$ & $\gamma$ & P. A. & & $\mathrm{N}$ & & $\mathrm{N}$ & & & & $\mathrm{x}$ & & & $\mathrm{N}$ & & & & & \\
\hline${ }^{133} \mathrm{Ba}$ & $\gamma$ & M. M. & & & A & A & & & & & & A & $\mathrm{N}$ & & & & & \\
\hline${ }^{140} \mathrm{Ba}$ & $\gamma$ & M. M. & & & A & & & & & & & & & & & & & \\
\hline${ }^{7} \mathrm{Be}$ & $\gamma$ & P. A. & & & & $\mathrm{N}$ & & & & & & & & & & & & \\
\hline${ }^{82} \mathrm{Br}$ & $\gamma$ & P. D. & & & & $\mathrm{X}$ & & & & & & & & & & & & \\
\hline${ }^{{ }^{141} \mathrm{Ce}}$ & $\gamma$ & P. A. & & & $\mathrm{N}$ & & & & & & & & & & & & & \\
\hline${ }^{143} \mathrm{Ce}$ & $\gamma$ & P. D. & & & $\mathrm{X}$ & & & & & & & & & & & & & \\
\hline${ }^{144} \mathrm{Ce}$ & $\gamma$ & P. D. & & & $\mathrm{X}$ & & & & & & & & & & & & & \\
\hline${ }^{58} \mathrm{Co}$ & $\gamma$ & M. M. & & & & A & & & & & & & & & & & & \\
\hline${ }^{60} \mathrm{Co}$ & $\gamma$ & M. M. & & & & A & & & & & & & W & & & & & \\
\hline${ }^{134} \mathrm{Cs}$ & $\gamma$ & M. M. & $\mathrm{A}$ & $\mathrm{N}$ & & A & $\mathrm{A}$ & $\mathrm{W}$ & A A & & & & $\mathrm{N}$ & & $\mathrm{A}$ & A A A & & \\
\hline${ }^{136} \mathrm{Cs}$ & $\gamma$ & M. M. & & & & A & & & & & & & & & & & & \\
\hline${ }^{137} \mathrm{Cs}$ & $\gamma$ & M. M. & A & $\mathrm{N}$ & A & $\mathrm{A}$ & A & A & A A & $\mathrm{N} \mathrm{N}$ & A & A & A & & A & A A A & & \\
\hline${ }^{152} \mathrm{Eu}$ & $\gamma$ & P. D. & & & & & & & & & & & & & & $\begin{array}{lll} & X & X\end{array}$ & & \\
\hline${ }^{59} \mathrm{Fe}$ & $\gamma$ & P. D. & & & & $\mathrm{X}$ & & & & & & & & & & & & \\
\hline${ }^{131} \mathrm{I}$ & $\gamma$ & M. M. & & & & A & & & & & & & & & & & & \\
\hline${ }^{132} \mathrm{I}$ & $\gamma$ & P. D. & & & $\mathrm{X}$ & & & & & & & & & & & & & \\
\hline${ }^{133} \mathrm{I}$ & $\gamma$ & P. D. & & & & $\mathrm{X}$ & & & & & & & & & & & & \\
\hline${ }^{40} \mathrm{~K}$ & $\gamma$ & M. M. & & & & & & & & $\mathrm{N} A$ & $\mathrm{X}$ & & A & A & $\mathrm{A}$ & & & \\
\hline${ }^{54} \mathrm{Mn}$ & $\gamma$ & M. M. & & & & A & $\mathrm{X}$ & & & & & & & & & & & \\
\hline${ }^{99} \mathrm{Mo}$ & $\gamma$ & P. D. & & & $\mathrm{X}$ & $\mathrm{N}$ & & & & & & & & & & & & \\
\hline${ }^{22} \mathrm{Na}$ & $\gamma$ & M. M. & A & $\mathrm{N}$ & & & & & A & & & & & & & & & \\
\hline${ }^{95} \mathrm{Nb}$ & $\gamma$ & P. D. & & & & $\mathrm{X}$ & & & & & & & & & & & & \\
\hline${ }^{147} \mathrm{Nd}$ & $\gamma$ & P. D. & & & $\mathrm{X}$ & & & & & & & & & & & & & \\
\hline${ }^{239} \mathrm{~Np}$ & $\gamma$ & M. M. & & & $\mathrm{A}$ & & & & & & & & & & & & & \\
\hline${ }^{210} \mathrm{~Pb}$ & $\alpha / \gamma$ & P. A. & & & & $\mathrm{N}$ & & & & & & & $\mathrm{N}$ & $\mathrm{N}$ & & & & \\
\hline${ }^{210} \mathrm{Po}$ & $a$ & P. D. & & & & & & & & & & & $\mathrm{X}$ & $\mathrm{X}$ & & & & \\
\hline${ }^{239+240} \mathrm{Pu}$ & $\gamma$ & P. D. & & & & & & & & $\mathrm{X}$ & & & & & & & & \\
\hline${ }^{86} \mathrm{Rb}$ & $\gamma$ & M. M. & & & & A & & & & & & & & & & & & \\
\hline${ }^{103} \mathrm{Ru}$ & $\gamma$ & M. M. & & & $\mathrm{W}$ & & & & & & & & & & & & & \\
\hline${ }^{124} \mathrm{Sb}$ & $\gamma$ & M. M. & & & & $\mathrm{W}$ & & & & & & & & & & & & \\
\hline${ }^{75} \mathrm{Se}$ & $\gamma$ & M. M. & & & & & & & & & & & & & & & A & A $\quad$ A \\
\hline${ }^{99 m} \mathrm{mc}$ & $\gamma$ & P. D. & & & $\mathrm{X}$ & $\mathrm{X}$ & & & & & & & & & & & & \\
\hline${ }^{65} \mathrm{Zn}$ & $\gamma$ & M. M. & A & & & 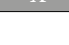 & & & & & & & & & & & & \\
\hline${ }^{95} \mathrm{Zr}$ & $\gamma$ & M. M. & & & $\mathrm{A}$ & $\mathrm{X}$ & & & & & & & & & & & & \\
\hline${ }^{238} \mathrm{U}$ & $a$ & P. A. & & & & & & & & $\mathrm{x}$ & & & $\mathrm{N}$ & $\mathrm{X}$ & & & & \\
\hline${ }^{235} \mathrm{U}$ & $\alpha / \gamma$ & P. D. & & & & & & & & $\mathrm{x}$ & & & $\mathrm{X}$ & $\mathrm{X}$ & & & & \\
\hline${ }^{234} \mathrm{U}$ & $\alpha$ & P. D. & & & & & & & & $\mathrm{x}$ & & & $\mathrm{x}$ & $\mathrm{X}$ & & & & \\
\hline${ }^{228} \mathrm{Ac}$ & $\gamma$ & M. M. & & & & & $\mathrm{A}$ & & A & $\mathrm{X}$ & & & $\mathrm{X}$ & A & $\mathrm{A}$ & & & \\
\hline${ }^{212} \mathrm{Bi}$ & $\gamma$ & P. A. & & & & & $\mathrm{X}$ & & A & & & & 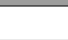 & $\mathrm{X}$ & A & & & \\
\hline${ }^{212} \mathrm{~Pb}$ & $\gamma$ & P. A. & & & & & $\mathrm{N}$ & $\mathrm{X}$ & A & $\mathrm{X}$ & & & $\mathrm{X}$ & A & $\mathrm{A}$ & & & \\
\hline${ }^{224} \mathrm{Ra}$ & $\alpha / \gamma$ & P. A. & & & & & $\mathrm{N}$ & $\mathrm{X}$ & A & $\mathrm{X}$ & & & $\mathrm{X}$ & A & A & & & \\
\hline${ }^{228} \mathrm{Ra}$ & $\alpha / \gamma$ & M. M. & & & & & A & A & A & $\mathrm{X}$ & & $\mathrm{W}$ & $\mathrm{X}$ & A & A & & & \\
\hline${ }^{228} \mathrm{Th}$ & $\alpha / \gamma$ & P. A. & & & & & $\mathrm{X}$ & $\mathrm{X}$ & A & $\mathrm{X}$ & & & $\mathrm{X}$ & $\mathrm{X}$ & $\mathrm{A}$ & & & \\
\hline${ }^{232} \mathrm{Th}$ & $a$ & P. D. & & & & & & & & $x$ & & & $\mathrm{X}$ & & & & & \\
\hline${ }^{208} \mathrm{Tl}$ & $\gamma$ & P. A. & & & & & $\mathrm{N}$ & $\mathrm{X}$ & A & $x$ & & & $\mathrm{X}$ & $\mathrm{W}$ & $\mathrm{A}$ & & & \\
\hline${ }^{214} \mathrm{Bi}$ & $\gamma$ & M. M. & & & & & A & $\mathrm{N}$ & & $\mathrm{N}$ & & & $\mathrm{x}$ & A & $\mathrm{A}$ & & & \\
\hline${ }^{234 \mathrm{~m}} \mathrm{~Pa}$ & $\gamma$ & P. D. & & & & & & & & $\mathrm{x}$ & & & $\mathrm{X}$ & & & & & \\
\hline${ }^{210} \mathrm{~Pb}$ & $\alpha / \gamma$ & P. A. & & & & & & & & $\mathrm{x}$ & $\mathrm{X}$ & & $\mathrm{X}$ & $\mathrm{N}$ & $\mathrm{A}$ & & & \\
\hline${ }^{214} \mathrm{~Pb}$ & $\gamma$ & M. M. & & & & & $\mathrm{A}$ & & & $\mathrm{N}$ & & & $\mathrm{X}$ & A & $\mathrm{A}$ & & & \\
\hline${ }^{226} \mathrm{Ra}$ & $\alpha / \gamma$ & M. M. & & & & & $\mathrm{A}$ & $\mathrm{N}$ & & $\mathrm{x}$ & & A & $\mathrm{X}$ & $\mathrm{A}$ & $\mathrm{A}$ & & & \\
\hline${ }^{234} \mathrm{Th}$ & $\gamma$ & P. D. & & & & & & & & $\mathrm{x}$ & & & $\mathrm{X}$ & & & & & \\
\hline & & & Cali & icación & LRA & & & Si cr & iterio d & de ponder & ación $>$ & $50 \%$ & & & M. M. & Madurez meto & doló & gica \\
\hline A & Acept & & & 3 & & & & $\mathrm{Si} \mathrm{cr}$ & iterio d & de ponder & ación $\mathrm{E}$ & ntre $20 \%$ & $6-50 \%$ & & P. A. I & En proceso y ce & on av & vances \\
\hline $\mathrm{W}$ & Aler & & & 2 & & & & $\mathrm{Si} \mathrm{cr}$ & iterio d & de ponder & ación & $20 \%$ & & & P. D. I & Por desarrollar & & \\
\hline $\mathrm{N}$ & No acep & & & 1 & & & & & & & & & & & & & & \\
\hline $\mathrm{X}$ & No repo & & & 0 & & & & & & & & & & & & & & \\
\hline
\end{tabular}


Los análisis de los esquemas de decaimiento en serie fueron evaluados resolviendo la ecuación general de Bateman con el empleo de la herramienta matemática MATLAB. En las figuras 3, 4 y 5 se presenta el resultado del modelamiento gráfico con la evaluación de tres (3) muestras objetivo que contienen emisores gamma de la serie natural del ${ }^{228} \mathrm{Ra}$. Esta herramienta permitió establecer un análisis comportamental y gráfico de lo que sucedió con los diferentes esquemas de decaimiento en serie.

Los resultados obtenidos para radionucleidos con emisiones por debajo de $150 \mathrm{keV}$ fueron en su mayoría no satisfactorios. Este hecho implica la necesidad de la mejora tanto en la calibración en eficiencia como en la corrección de la autoabsorción, al estar estas energías muy influidas por el efecto fotoeléctrico [9], [10]. La complejidad de estas correcciones implica el establecimiento de un método de cálculo para corregirlas en un futuro y dentro de las posibilidades del laboratorio. El uso de materiales de referencia con similares densidades y composición química es lo más adecuado para esta actividad [11]; sin embargo, es un proceso que implica tiempo, por lo que el LRA debe establecer un método adecuado para su manejo; dentro de las posibilidades se encuentra la aplicación de programas matemáticos.

En la tabla 4, la segunda columna (acompaña a cada radionucleido) muestra el resultado del criterio de aceptación de la ponderación. De igual manera se presentan resumidos los resultados de las calificaciones obtenidas para cada radionucleido, conforme el modelo de calificación llevado a cabo por el programa de evaluación de desempeño ALMERA. Se tienen 24 radionucleidos con una calificación de $m a$ durez metodológica, celda color verde; 11 radionucleidos con calificación de en proceso y con avances, celda color amarillo; y 18 radionucleidos con una calificación de por desarrollar. Las principales causas de las desviaciones identificadas para estos radionucleidos, tanto artificiales como naturales, acorde a la calificación del programa ALMERA y la ponderación interna del LRA, se enfocaron en:

Asegurar equilibrio secular entre la madre y sus hijas.

Asegurar el selle hermético de las muestras en las diferentes matrices.

Contar con MRC con el mismo contenido isotópico para la calibración de eficiencia en detectores de germanio, respecto a los identificados durante la evaluación.

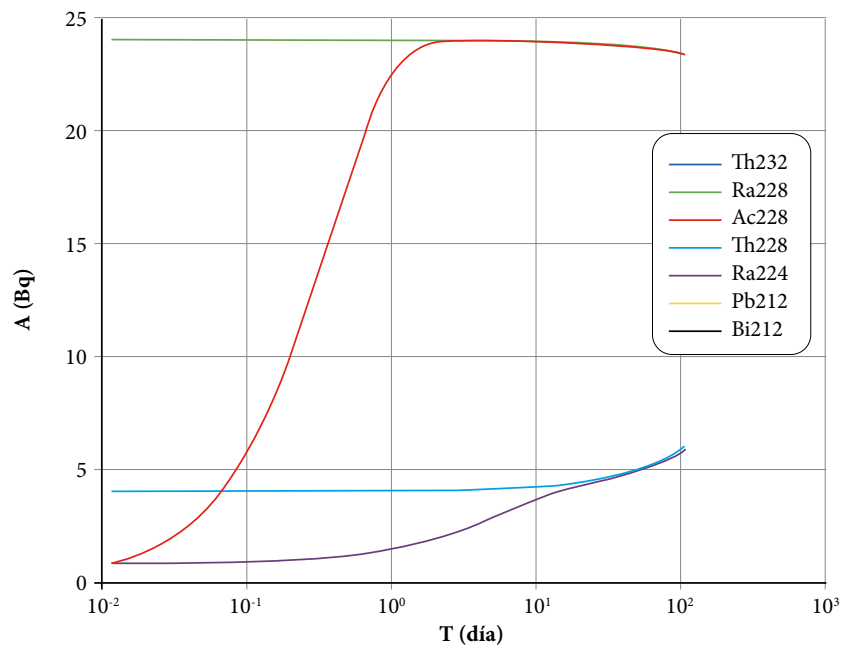

Figura 3. Comportamiento serie natural ${ }^{232} \mathrm{Th}$ en matriz líquida. Herramienta $M A T L A B$

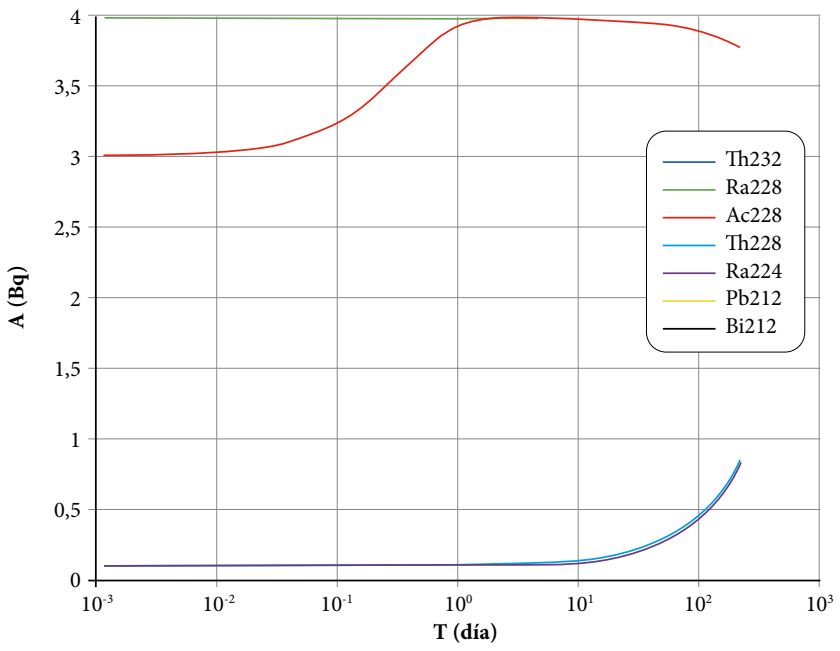

Figura 4. Comportamiento serie natural ${ }^{232} \mathrm{Th}$ en matriz líquida. Herramienta MATLAB

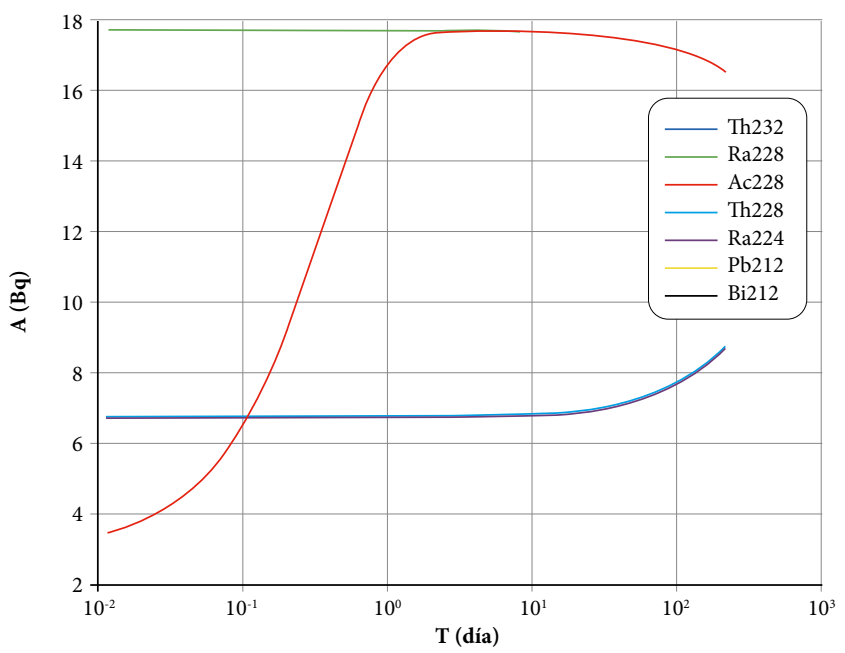

Figura 5. Comportamiento serie natural ${ }^{232} \mathrm{Th}$ en matriz sólida. Herramienta MATLAB 
Tener una calibración adecuada y eficiente a bajas energías, evaluando la necesidad de corregir correctamente por el efecto fotoeléctrico.

Mantener un control de blancos y su uso adecuado, conforme la densidad de la muestra.

» Asegurar el cumplimiento de los límites de detección (LID) definidos técnicamente para los radionucleidos de interés institucional.

\section{Conclusiones}

La participación en los ejercicios de intercomparación ha posibilitado la evaluación del desempeño del laboratorio, y ha permitido tanto establecer los parámetros operacionales necesarios para la determinación de bajas concentraciones de emisores gamma en matrices ambientales, como identificar y cuantificar radionucleidos por medio de una respuesta rápida, en cumplimiento de los límites de detección (LID) establecidos para cada radionucleido.

El LRA logró mejorar la calificación del desempeño a través de la implementación rigurosa y metódica de los controles internos (energía, resolución, eficiencia), mejorando la definición de los criterios operacionales (LID, tiempo de medida, geometría, alícuota de la muestra).

Las actividades de entrenamiento de los profesionales del LRA, en espectrometría gamma, las correcciones por decaimiento, las correcciones de la eficiencia, el uso de la ecuación de Bateman, así como la conceptualización de soportado y no soportado incidieron satisfactoriamente en la mejora de la calificación del desempeño del LRA en los ejercicios de intercomparación.

Las mejoras logradas en los últimos años en el LRA ofrecen confianza en los resultados reportados para la determinación de radionucleidos donde la madurez metodológica ha sido alcanzada.

\section{Referencias}

[1] I. Canberra Industries, Model S574 LabSOCS ${ }^{\mathrm{TM}}$ Calibration Software, Technical Reference Manual, Meriden: Canberra Industries, Inc., 2015.

[2] R. Eva, "Aplicaciones de la simulación Monte Carlo en dosimetría y problemas de física médica”, de Contribuciones del Instituto Nacional de Investigaciones Nucleares al avance de la Ciencia y la Tecnología en México, Ocoyoacac, Instituto Nacional de Investigaciones Nucleares al avance de la Ciencia y la Tecnología en México, 2010, pp. 89-105.

[3] International Organization for Standardization, NTC-ISO/ IEC17025:2017, Requisitos generales para la competencia de los laboratorios de ensayo y calibración, Bogotá, 2017.

[4] International Union of Pure and Applied Chemistry, "Harmonized Guidelines for Single - Laboratory Validation of Methods of Analysis", Pure and Applied Chemistry, vol. 74, n. ${ }^{\circ}$ 5, pp. 835-855, 2002. https://doi. org/10.1351/pac200274050835

[5] A. Luca, A. Watjen, E. Grigorescu, M. Sahagia y C. Ivan, "Conclusions from the participation at proficiency test for gamma-ray spectrometry measurements", Romanian Journal of Physics, vol. 55, n. ${ }^{\circ}$ 7-8, pp. 724-732, 2010.

[6] R. Venkataraman, F. Bronson, V. Atrashkevich, M. Field y B. M. Young, "Improved detector response characterization method in ISOCS and LabSOCS”, Journal of Radioanalytical and Nuclear Chemistry, vol. 264, n. ${ }^{\circ}$ 1, pp. 213219, 2005. https://doi.org/10.1007/s10967-005-0696-7

[7] F. L. Bronson, "Validation of the accuracy of the LabSOCS software for mathematical efficiency calibration of Ge detectors for typical laboratory samples", Journal of Radioanalytical and Nuclear Chemistry, vol. 255, n. ${ }^{\circ}$ 1, pp. $137-$ 141, 2003. https://doi.org/10.1023/A:1022248318741

[8] S. I. Kafala, "Simple maethod for true coincidence summing", Journal of Radioanalytical and Nuclear Chemistry, vol. 191, n. ${ }^{\circ}$ 1, pp. 105-114, 1995. https://doi.org/10.1007/BF02035990

[9] A. Barba-Lobo, F. Mosqueda y J. Bolívar, "A general function for determining mass attenuation coefficients to correct self-absorption effects in samples measured by gamma spectrometry", Radiation Physics and Chemistry, vol. 179, pp. 1-11, 2021. https://doi.org/10.1016/j.radphyschem.2020.109247

[10]G. R. Gilmore, Practical Gamma-ray Spectrometry, Warrington: John Wiley \& Sons, Ltd, 2008.

[11]Z.-N. Tian, X.-P. Ouyang, Y. Liu, L. Chen, J.-L. Liu, X.P. Zhang, J.-W. Song y M. Zeng, "Self-attenuation corrections calculated by LabSOCS Simulations for gamma-spectrometric measurements with HPGe detectors", Chinese Physics C, vol. 38, n. ${ }^{\circ}$, pp. 1-6, 2014. https://doi. org/10.1088/1674-1137/38/7/076002 